\title{
The Logic and Fundamental Concepts of the Grand Unified Theory*
}

\author{
Edgar E. Escultura \\ Research Professor (Honorary), GVP_-Prof. V. Lakshmikantham Institute for Advanced Studies, GVP College of \\ Engineering J. Nehru Technical University Kakinada, Vishakapatnam, AP, India \\ Email: escultur36@gmail.com, http://users.tpg.com.au/pidro
}

Received May 27, 2013; revised July 9, 2013; accepted August 6, 2013

Copyright (C) 2013 Edgar E. Escultura. This is an open access article distributed under the Creative Commons Attribution License, which permits unrestricted use, distribution, and reproduction in any medium, provided the original work is properly cited.

\begin{abstract}
The grand unified theory (GUT) originated in mathematics with this question: why are there long standing unsolved problems in mathematics, e.g., Fermat's conjecture (also known as Fermat's last theorem (FLT))? The answer came quickly: its underlying fields - foundations and the real number system - are defective. In particular, formal logic is inapplicable to mathematics (language of science) and the real number system is inconsistent. Critique-rectification of these fields was undertaken leading to a new mathematical methodology and the consistent new real number system that provides the main mathematics of GUT. Similar question was posed in physics: why are there long standing problems, e.g., the gravitational n-body and turbulence problems? The answer: the present methodology, quantitative modeling is inadequate and the remedy is a new methodology — qualitative mathematics and modeling - that solved these problems and provided the initial formulation of GUT. This paper presents the basic logic of GUT and its fundamental concepts, particularly, the superstring or fundamental building block of matter.
\end{abstract}

Keywords: Anti-Matter; Big Bang; Black Hole; Chaos; Cosmic Burst; Cosmological Body; Electromagnetic Wave; Generalized Physical Fractal; Qualitative Modeling; Quantum and Macro Gravity; Quark; Primum; Superstring; Turbulence

\section{Introduction}

This expository paper reconstructs the logic of the grand unified theory (GUT) [1] and its formulation and fundamental concepts, a journey that started with this question: why are there long standing unsolved problems of mathematics, e.g., the 355-year-old Fermat's conjecture (also known as Fermat's last theorem (FLT) [2])? The answer: its underlying fields - foundations and the real number system-are defective [3]. Formal logic is inapplicable to mathematics as language of science and the latter is inconsistent [3]. The remedy: the new methodology of qualitative mathematics and construction of the consistent new real number system that resolves FLT $[4,5]$ and provides the main mathematics of GUT [6].

Similar question was raised in physics: why are there long standing unsolved problems, e.g., the gravitational n-body and turbulence problems [6,7]? The answer: the present methodology of quantitative modeling (formerly called mathematical modeling [8]) that describes nature and natural phenomena mathematically is inadequate.

"Logic refers to scientific reasoning defined by qualitative modeling.
Remedy: the new methodology, qualitative mathematics and modeling (QMAM), that explains nature and natural phenomena in terms of natural laws introduced in and the main contribution of [9] that solves both problems and provides the strategy for the development of GUT. The catalyst was the n-body problem that says:

Given n-bodies in the Cosmos of masses $m_{1}, m_{2}, \cdots, m_{n}$, initial positions $x_{1}, x_{2}, \cdots, x_{n}$, velocities $v_{1}, v_{2}, \cdots, v_{n}$, and initial time T subject to mutual gravitational attraction between them, find their positions, velocities and trajectories at later time.

Simon Marquiz de Laplace posed this problem at the turn of the 18th century to prove the stability of the solar system which was then thought to be the universe. In this context, we take the $\mathrm{n}$ bodies as cosmological bodies but we do not know what a body is, where it comes from, what gravity is and how it determines their motion. Most of all, we cannot use differential equations since the boundary conditions belong to the past. Therefore, we need to know the fundamental building block of matter, cosmology, gravity and its effect on the bodies. The solution came in 1997 [6]. 


\section{The New Methodology}

Among the relevant important rectification of and requirements on foundations and the real number system are [3,4]: 1) A mathematical system or space consists of objects in the real world called concepts, e.g., symbols, words and figures, subject to consistent basic premises or axioms; 2) The rules of inference for making conclusion follow from the axioms; and 3) every concept is defined by the axioms, i.e. undefined term is inadmissible for it brings in ambiguity. The new real number system is built on three consistent axioms [4] instead of the inconsistent field axioms [10].

As in mathematics, the n-body problem requires construction of physical theory that provides the solution. A physical theory is a mathematical space where the axioms are laws of nature. Only QMAM whose mathematical component-qualitative mathematics-models rational thought qualitatively is capable of building physical theory, particularly, GUT. Qualitative mathematics involves the following activity:

Making conclusions, visualizing, abstracting, thought experimenting, learning, creating abstract and physical concepts (the former created by thought, the latter has referent), intuition, imagination, trial and error to sift out what is valid, negating what is known to gain insights into the unknown, altering premises or axioms to draw out new conclusions, thinking backwards, finding basic premises for a mathematical space and devising techniques that yield results.

Qualitative mathematics includes abstract mathematical spaces and the search for the natural laws.

\section{Physical Concepts and Natural Laws}

We build GUT as physical theory and since our universe is made up of the same fundamental building block we cannot separate quantum from macro gravity so that, true to its name, GUT unifies the natural sciences with this common building block under this methodology. Macro gravity encompasses astrophysics and cosmology and quantum gravity quantum physics and its extension to dark matter. They are pillars of GUT [1] the other pillar thermodynamics in the broad sense (extended to dark matter). The existence, structure, properties and behavior of every physical concept is defined by natural laws but mathematical concepts are created by thought, e.g., time, distance, and have no physical referents.

We partially define physical concepts until fully defined by natural laws. Energy is motion of matter; therefore, matter and energy are never separate and anything that has energy, e.g., photon, has mass and vice versa. Wave is suitably synchronized sequence of resolution of contending forces in the medium, i.e., suitably synchronized vibration [11]. Dark matter, one of the two fundamental states of matter, consists mainly of non-agitated but also semi-agitated superstrings; it is not directly observed but known only through its impact on visible or ordinary matter, the other fundamental state.

Basic cosmic or electromagnetic wave is generated by natural vibration of atomic nucleus propagated across dark matter that fills up the Cosmos. Nuclear vibration is due to the impact of electromagnetic waves coming from all directions its characteristics determined by the nuclear structure in accordance with this natural law [11]:

Internal-External Factor Dichotomy Law. The interaction, dynamics and physical characteristics of a physical system are shaped by internal and external factors; in general the internal is principal over the external and the latter works through the former.

The characteristics of electromagnetic wave are determined by the generating nuclear vibration. Flux is motion of matter with identifiable direction at each point, e.g., water current. Turbulence is coherent flux, e.g., typhoon, wave. Chaos is mixture of order none of which is identifiable. For example, at the onset of hurricane air molecules rush by the trillions towards tropical depression; the motion of a molecule cannot be predicted due to the immensity of the quantity of rushing molecules and collisions (uncertainty of large number $[3,4]$ ). However, every molecule is subject to natural laws that define its order.

\section{The Grand Unified Theory}

The development of GUT requires identification of the fundamental natural law, finding others consistent with it and if inconsistency with a natural phenomenon arises, we find another natural law that reconciles them. The latter exists because of the order of our universe defined by natural laws. A physical theory is valid if it explains natural phenomena, prediction based on it is verified and technology based on it works.

\subsection{Quantum Gravity}

The most fundamental natural law is an enrichment and extension of the first law of thermodynamics:

Energy Conservation Law. In any physical system and its interaction, the sum of kinetic (visible) and latent (dark) energy is constant, gain of energy is maximal and loss of energy is minimal.

The next natural law identifies various expressions of Energy Conservation.

Energy Conservation Equivalence Law. Energy conservation has many expressions or forms: order, symmetry, economy, least action, optimality, efficiency, stability, self-similarity (nested fractal), coherence, resonance, quantization, synchronization, smoothness, uniformity, motion-symmetry balance, non-redundancy, non-extravagance, evolution to infinitesimal configuration, helical and related configuration such as circular, spiral, sinusoi- 
dal and a loop, in biology, genetic encoding of characteristics, reproduction and order in diversity and complexity of functions and configuration that provides optimal capability.

We call each component of this law physical principle. The next natural law was inspired by a high school experiment many years ago.

Flux-Low-Pressure Complementarity Law. Low pressure sucks matter around it and the initial rush of matter towards a region of low pressure stabilizes into local turbulence, e.g., vortex flux; conversely, coherent flux induces low pressure around it.

The next law is central to primal and cosmological ininteractions. We first state its broad form.

Flux Compatibility*. Two fluxes of the same direction attract but two fluxes of opposite directions repel each other.

Matter forms steadily in the Cosmos as dust clouds that congeal into stars at the rate of one star per minute and star nurseries where stars form rapidly [12-14].

With these findings and by Energy Conservation dark matter exists and the question "what does it consist of?" is legitimate, i.e., non-vacuous. Our answer is: the superstring, fundamental building block of our universe or nature; we embellish it with structure, properties, etc.

What are the requirements? It must be indestructible; otherwise, our universe would have collapsed a long time ago, and unique, i.e., like the electron, there is only one superstring with unique structure, properties, etc. Uniqueness follows from being the fundamental building block.

The only force that interacts with it is electromagnetic wave. When hit by suitable electromagnetic wave a nonagitated superstring (a) is thrown by its impact, bounces with others and comes to rest as dark matter when the imparted energy dissipates or (b) gets close to its earlier path, is sucked by it, by Flux-Low-Pressure Complementarity, and forms a loop, the original non-agitated superstring called toroidal flux traveling through the loop at $7\left(10^{22}\right) \mathrm{cm} / \mathrm{sec}$ [15]. By Energy Conservation and Energy Conservation Equivalence, its path shrinks and evolves to energy-conserving form: circular helical loop, its toroidal flux traveling through its cycles at this speed. By the fractal principle its toroidal flux, being a superstring, has toroidal flux, a superstring, travelling at this speed, etc., leading to formation of fractal sequence of toroidal fluxes, each a superstring, without a last element. The first term of the sequence is a semi-agitated superstring. The superstring is identified with the first term of its fractal sequence since its interactions are determined by it. We summarize our findings as a natural law.

Existence of Basic Constituent of Matter and Its Generalized Nested Fractal Structure. The basic constituent of dark matter is the non-agitated superstring, a circular helical loop and nested fractal sequence of su- perstrings or toroidal fluxes, with itself as first term; each toroidal flux in the sequence is a superstring having toroidal flux, a superstring, traveling at $7\left(10^{22}\right) \mathrm{cm} / \mathrm{sec}$ through its cycles, etc.; each superstring except the first, is contained in and self-similar to the preceding term in structure, behavior and properties.

This structure is nested generalized physical fractal $[16,17]$. The first term of the fractal sequence looks like a lady's spring bracelet (figure in [18]). Self-similarity means that each term in the fractal sequence except the first is similar to the preceding in structure and properties, in this case, being helical circular loop with toroidal fluxa superstring - traveling through it at this speed.

By the quantization and synchronization principles, this speed of $7\left(10^{22}\right) \mathrm{cm} / \mathrm{sec}$ is a constant of nature, e.g., speed of electric current. There is another possibility: (c) hit by suitable electromagnetic wave the first term of a non-agitated superstring expands and becomes semiagitated, by Energy Conservation. In both cases (a) and (b) the superstring is a generalized nested physical fractal sequence of superstrings. Its latent energy comes from the motion of its toroidal fluxes, a super, super huge amount due to its fractal sequence structure.

When suitable electromagnetic wave hits a semi-agitated superstring a pair of mutually exclusive events occurs: (d) the first term of its fractal superstring bulges to retain the toroidal flux speed despite the energy imparted by the electromagnetic wave, by Energy Conservation, turning it into an agitated superstring called primum, unit of visible matter, its toroidal flux non-agitated, or (e) the first term breaks, its toroidal flux remaining non-agitated.

A superstring is non-agitated if its cycle length (CL) is less than $10^{-16}$ meters, semi agitated if $10^{-16}<\mathrm{CL}<10^{-14}$ meters and agitated if a segment has $\mathrm{CL}>10^{-14}$ meters.

The next law articulates our findings.

Dark-to-Visible-Matter Conversion. When suitable electromagnetic wave hits a semi-agitated superstring one of these occurs: (a) the outer superstring breaks, its toroidal flux remaining non-agitated superstring; (b) a segment bulges into a primum, an agitated superstring and a unit of visible matter.

When electromagnetic wave hits a superstring at most the first of its fractal sequence of superstrings breaks leaving the rest intact and nested fractal sequence of superstrings, i.e., a superstring. Thus, this structure insures indestructibility. It follows that the Universe of dark matter has no beginning and no end (timeless). Moreover, by Flux-Low-Pressure Complementarity, it has no boundary, i.e., unbounded and infinite and our universe is a finite local bubble in it among other universes [19-21]. This does not rule out the possibility that the set of local universes is also unbounded and infinite.

A physical system is observable through the medium of light if its size is comparable with the wavelength of 
light; this follows from the Resonance Law [22,23]. Thus, a semi- or non-agitated superstring is not observable its cycle length being less than the finest wavelength of visible light in order of magnitude, $10^{-14}$ meters.

What really is a primum? When the toroidal flux along its cycles is hit by cosmic waves coming from all directions it is thrown into erratic motion and collides with other superstrings turning it into a spike with the centroid traveling through the cycles at $7\left(10^{22}\right) \mathrm{cm} / \mathrm{sec}$. It pulls the superstrings around the primum into its induced vortex flux with axis coinciding with the axis of the primum inside the cylindrical eye making it a magnet, polarity in accordance with the right hand rule of electromagnetism (Figure 2 of [24]). The induced vortex flux is counterclockwise for a positive primum, by convention, negative otherwise. The plane that passes through the apex of its profile and normal to its axis is the equatorial plane its intersection with the primum the equator.

The induced vortex flux manifests itself as charge. The unit of charge is the electron's charge: $-1\left(1.6\left(10^{-19}\right)\right.$ coulombs). The electron, +quark and -quark having charges $-1,2 / 3$ and $-1 / 3$ [25], respectively, are basic prima because they comprise the atom. Seismic waves generated by the micro component of turbulence at the spinning core of a cosmological vortex [7,21] also convert dark to visible matter in and around it at staggering rate.

The primum's toroidal and induced vortex flux and natural vibration of dark matter endow dark matter huge latent energy partially convertible to kinetic energy. The latent energy density of dark matter is $10^{26}$ joules/cubic $\mathrm{ft}$. according to de Broglie [26], $8\left(10^{8}\right)$ volts/cm says Seike Jr. [26] and the equivalent of $18 \mathrm{~kg} / \mathrm{cu}$ meter according to Gerlovin [25] using relativistic conversion.

The next natural law is a special form of Flux Compatibility ${ }^{*}$ that applies to vortex fluxes of superstrings in quantum and macro gravity directly.

Flux Compatibility. Two prima of opposite toroidal flux spins attract at their equators but repel at their poles; otherwise, they repel at their equators but attract at their poles. Two prima of same toroidal flux spin connect equatorially only through a primum of opposite toroidal flux spin between them called connector.

\subsubsection{Primal Interaction}

Primal interaction is governed by Flux Compatibility and Flux-Low-Pressure Complementarity.

The proton consists of two +quarks joined by a -quark at their rims, by Flux Compatibility (Fig. 6(a), [24]), their axis coplanar, by Energy Conservation, and its charge: $2 / 3-1 / 3+1 / 3=1$. Thus, the proton has counter-clockwise vortex flux spin. Since a simple primum is charged, the neutral neutrino is a coupled pair of simple prima of numerically equal but opposite charges, say, $+\mathrm{q}$ and $-\mathrm{q}$, so that its charge is $+q+-q=0$, neutral [27].
By Flux Compatibility, stability and optimality, the electron attaches to both +quarks beside the -quark but away from the negative quark in the proton, by Flux Compatibility, their centers viewed from the north pole form the vertices of a quadrilateral. Their coherent fluxes make its interior a region of low pressure that sucks only light neutral primum (since charged primum is repelled by charged primum already in the coupling). This is the configuration of the neutron and its charge: $+2 / 3-1 / 3+$ $2 / 3-1+0=0$, i.e., neutral (Fig. 6(b), [24]).

Since the masses and composition of these prima are known [27] we compute the neutrino's mass:

1) neutron: $1.674\left(10^{-27}\right) \mathrm{kg}$; proton: $1.672\left(10^{-27}\right) \mathrm{kg}$; electron: $9.611\left(10^{-31}\right) \mathrm{kg}$.

Converting to atomic mass unit (amu) their masses are:

2) neutron: $1.0087 \mathrm{amu}$; proton: $1.0073 \mathrm{amu}$; electron: 5.486 $\left(10^{-8}\right) \mathrm{amu}$, and neutrino's mass is: $\eta=8.5\left(10^{-8}\right)$ amu, 1.55 times electron's mass (latter's mass 1840 times proton's mass).

\subsubsection{The Atom, Molecule, Heavy Isotope}

The protons are first to form the nucleus of the atom (Fig. $7,[24])$. When there is only one proton it coincides with the eye of its vortex flux. If there are more their vortex fluxes add up to form the vortex flux around it the protons which are joined pairwise by -quarks (their arrangement discussed in [28]). Clearly, the nucleus is fractal; therefore, electromagnetic waves it generates is fractal and endowed with huge energy.

As positive coupled primum, the nucleus is a magnet of positive polarity with the vortex flux around it providing the magnetic field. Viewed from the north-pole the vortex flux of a free atom spins counterclockwise (right hand rule of electromagnetism).

The electrons being negatively charged are attracted to the vortex flux away from the eye but being light, they are swept into orbit by the vortex. By centrifugal force, the most energetic orbital electrons are those closest to the equatorial plane; they form the outermost subshells [28]. The least energetic ones cluster near the poles and form the lowest orbital shells. A stable atom has orbital electrons equal to the number of protons in the nucleus. Otherwise, it is a positive or negative ion and reacts with other prima. Moreover, the eye sucks non-agitated superstrings that accumulate steadily in the nucleus as mini black hole [28], the principal source of nuclear energy in nuclear fission. In fact, every charged primum, simple or coupled, sucks and accumulates mini black hole in the eye. This was confirmed for the proton at CERN [29] by great burst of energy attributed to the Higgs boson released when two protons collide at great speed.

The neutron is the only primum sucked by the nuclear eye to form heavy isotope (see [28] for arrangement). 
The usual molecular formation has one valence electron, a -quark, each from two atoms at their outer subshells that serve as connector [28] so that the molecule has two electron connectors.

\subsection{Macro Gravity}

Macro gravity is the science of our universe as super, super galaxy; we focus on cosmology - its birth, evolution, destiny and physical systems at macro scale, e.g., galaxies. It is a local "bubble" in the boundless, timeless Universe of dark matter. This is a new perspective where previously it was thought our universe was the Universe, a source of error in the estimate of its age [1], especially, with the discovery of stars in the Milky Way older than the Big Bang [30,31].

\subsubsection{Ordinary Universe}

By energy conservation the superstrings shrink steadily and. by the law of uneven development depressions form each containing depressions, etc. Therefore, they form nested fractal sequences of depressions with the original depression the common first term. By Flux-Low-Pressure Complementarity each depression becomes the common first term of nested fractal sequences of cosmological vortices of superstrings around it. It becomes the major cosmological vortex whose eye sucks and pulls the surrounding fractal sequences of cosmological vortices along rotating spirals falling towards and winding around and merging with the spinning collected mass around the eye called the core, a cosmological body. Its equatorial plane passes through the center of the eye normal to its axis.

The spinning discular halo of vortex flux of visible matter containing all cosmological bodies called minor cosmological vortices and their cores called minor cosmological bodies under its influence including the spirals of falling cosmological vortices and cosmic dust is discular [32] just as its gravitational flux that wraps it is. The gravitational flux determines the internal dynamics of this cosmological vortex including gravity which is the suction by the eye. The same dynamics, processes and structure are replicated in each of the minor vortices, e.g., star and planet in the case of a galaxy.

Initially, a cosmological vortex is dark but its tremendous spin (kinetic energy) agitates and converts the superstrings around the eye to visible matter, first as simple prima and then as light elements. The bulk of the conversion is due to its micro component of turbulence [7,21] in the inner core that generates seismic waves and convert dark to visible matter in the core and around it [21]. In a galaxy the increasing power of spin combined with dark viscosity results in: (a) stretching of suction by the eye and reach of the rotating spiral paths of falling minor vortices and their cores (seen in young galaxies called spiral nebulae [32] at the ascent phase of their development) and (b) increasing centrifugal force on the revolving cosmological bodies along the spirals.

The balance between the suction by the eye (gravity) and centrifugal force on the rotating cosmological bodies is attained first on the periphery of the vortex flux along the equatorial plane that puts the bodies there into orbit around the eye. The locus of this point approaches the eye along the spiral so that all visible bodies near the core are engulfed by gravity first. Mercury was the last planet to escape the Sun's gravity.

\subsubsection{Evolution of Cosmological Vortex}

The expansion and increasing power of a major vortex continues until it gets pulled by an even more powerful cosmological vortex of opposite vortex spin, if any, and becomes the latter's minor vortex. Vortex fluxes of the same spin along a common equatorial plane repel each other but when their equatorial planes are oblique to each other they can collide. Such an event between two galaxies was observed in 1995 [20].

Interaction between cosmological vortices leads to formation of maximal major vortices, by the quantization principle. When a maximal cosmological vortex has a galaxy as minor cosmological vortex we call it an ordinary universe.

\subsubsection{Example of Ordinary Universe}

Milky Way was an ordinary universe since it has 10 galaxies as minor cosmological vortices [32] before it was pulled into orbit by our universe which is a special universe. So does Andromeda have 22 galaxies as minor cosmological vortices [32] but it probably formed in our universe because it is quite young as shown by its bright and robust spirals of falling stars [328]. The discovery of stars in the Milky Way older than the Big Bang shows that it is older than our universe [31]. This explains its faint uneven spirals of falling matter revealing that much of its visible matter has been sucked by gravity. Moreover, we can see the Cosmic Burst (Second Biggest Bang [33]) from our vantage point proving Milky Way was far from the Big Bang when it occurred 8 billion years ago and was not part of our young universe [21].

Milky Way has 400 billion stars [32,34]. Its visible discular halo along its galactic equatorial plane is 100 million light years across, its crater 10 million light years thick $[32,34]$. Sagittarius, relic of a former galaxy and now a cloud of stars has been "cannibalized" by Milky Way that has gobbled up some of its stars [32].

Andromeda's visible discular halo is 200 million light years across; has mass equivalent to 3500 billion Suns $[32,34]$; has a double core at the center indicating it almost formed a binary galaxy; has discular halo of a galaxy due to centrifugal force, thick at the center where 
visible matter collects due to suction by the eye but thin at the rim along the equatorial plane due to stretching by centrifugal force. Two of Andromeda's 22 minor galaxies are at opposite sides of and near its visible discular halo and appear headed for gravitational gobbling [32].

This dynamics of the evolution of a cosmological vortex applies to our universe as a super, super galaxy.

\subsubsection{Cosmological Vortex Interaction}

In any cosmological vortex the lucky few minor vortices at the balance between suction by the eye and centrifugal force take their orbits around the eye along rotating spirals. In the solar system they are the planets and planetoids that orbit the Sun. The Sun is a minor cosmological vortex of the Milky Way and what we see is its visible core.

Consider any cosmological vortex. Since it rotates at great speed, greatest at the equator and 0 at the poles, centrifugal force throws visible matter outward at the equator. Then it becomes a thin disc of visible matter consisting of minor vortices and their cores and clouds of cosmic dust riding on the gravitational flux which is thick and concentrated around the eye. The discular cosmological vortex is seen in image of a galaxy.

Vortex flux spin determines interaction between cosmological vortices mediated by their gravitational fluxes in view of Flux Compatibility: two vortices of opposite spins are attractive at their rims along their equatorial planes; they are repulsive otherwise. If they have the same spin and their masses have the same order of magnitude, they evolve into binary cosmological vortices each revolving around the other and mutually riding on each other's spiral flux; centrifugal force prevents them from crushing into each other. If they have the same spin, regardless of their relative masses, they have mutual repulsion unless one is a giant compared to the other in which case the more massive one gobbles up the other by gravity. However, if one is large compared to the other and has opposite spin, the latter rides as minor vortex or an eddy on the gravitational flux towards and merges smoothly with the core of the former unless the centrifugal force on the smaller vortex balances the main gravitational flux pressure in which case it takes elliptical orbit around the main core. Otherwise, if centrifugal force exceeds gravitational pull on a body, it gets catapulted off the vortex's influence along the equatorial plane. This is what happened to the galaxy clusters traversing our universe [19].

Elliptical orbit, being due to radial oscillation is the most probable orbital configuration since perfect balance that yields circular orbit is unstable, by Uneven Development. A minor vortex along the main spiral streamline that spins opposite that of the main vortex either forms elliptical orbit around it as eddy or gets sucked into and is crushed by the core and joins it. As an eddy a vortex has relative autonomy. Two contiguous vortices of comparable masses with the same spin do not crash into each other due to mutual repulsion of opposite fluxes, another case of quantum-macro gravity duality.

\subsubsection{The Destiny of a Cosmological Vortex}

The steady suction by the eye thins out both dark and visible matter and weakens the impact of both gravity and spin, again, starting from the rim of the equatorial disc where gravity is weakest and moving on towards the core until the core vortex is completely isolated from the minor vortices and the latter, in turn, become independent cosmological vortices like the cloud of stars of Sagittarius. Over a long period of time the core spin considerably weakens and much of the primal bonding declines so that the core becomes mainly a cluster of individual prima at the boundary of the eye or event horizon bound together only by the eye's suction. At this time the core, in the case of a star, has lost so much energy that it does not radiate much energy anymore its spin almost ground to a halt. This phase is referred to as a neutron or dwarf star (nothing to do with the neutron). There are many such stars in the Cosmos and massive ones each as much as 200 million times the mass of our Sun. They may have been the cores of earlier small galaxies. Eventually, they convert to semi-agitated superstrings at the event horizon. Thus, a neutron star is the transitional phase of a star to its destiny as black hole.

Since the eye is a region of calm it de-agitates the weakened core around it layer by layer until its constituent superstrings become non-agitated and collect in the eye as black hole, massive concentration of non-agitated superstrings. By the principle of uniformity, every cosmological vortex including the Earth has a black hole in the eye $[21,35,36]$.

Contrary to popular belief, a black hole does not suck matter around it. It is the eye of a cosmological vortex that nurtures and builds it that does. A completed black hole is necessarily naked and there are many such black holes in the sky marked by absence of visible matter.

\subsubsection{Our Universe}

Our universe traces its origin to the Big Bang, a rare colossal event, an explosion of a super, super massive black hole 8 billion years ago [1,21,30], destiny of the core of a previous special universe $[21,30]$. The Big Bang was caused by a sequence of hits on that black hole by electromagnetic waves that triggered chain reaction and eventual explosion. It created a super ... super depression that started the formation of a super, super galaxy which has now evolved to our universe [21,37].

The Big Bang created shock waves (highly energetic nested fractal cosmic waves) that pushed the Cosmic 
Sphere outward into accelerated expansion while at the same time being pushed by dark matter exterior to it due to suction by the super, super depression. Thus, the trapped superstrings were compressed between the inner and outer boundaries (layers) of the Cosmic Sphere by the two opposite forces. The most energetic shock waves pierced through the Cosmic Sphere and converted dark to visible matter in its immediate vicinity. The less energetic ones bounced between the outer and inner boundaries agitating the trapped superstrings but only up to semiagitated superstrings due to compression.

Continued expansion combined with outward pressure from the compressed semi-agitated superstrings weakened it until it burst at $t=1.5$ billion years from the start of the Big Bang (Cosmic Burst) [33] much more powerful than the Big Bang due to the great infusion of energy during 1.5 billion years of semi-agitation of the trapped superstrings. We put the birth of our universe at this point because the released semi-agitated superstrings converted to prima in the neighborhood of the once Cosmic Sphere and formed bright radioactive clusters called quasars consisting mainly of simple, therefore, charged prima, the initial visible matter of our early universe. They peaked at $t=2.5$ billion years [35]. As the temperature cooled coupled prima and light elements formed and got entangled into the galaxies around it which, in turn, were drawn to it by gravity and became minor vortices. Thus, the Big Bang did not create new galaxies other than our early universe that evolved into our universe.

As our universe increased its spin it imparted greater centrifugal force on the galaxies but suction by the eye balanced it and induced them to form elliptical orbits around it, by Uneven Development. As its power rose further, centrifugal force surpassed gravitational suction and catapulted galaxies outward. This explains its present accelerated radial expansion [38,39] which has nothing to do with the Big Bang anymore. As our early universe continued to expand due to increasing centrifugal force on the galaxies that have been drawn into it its gravitational pull on the galaxies around it broadened.

Our universe is not the only special universe; the galaxy clusters traversing our universe [33] must have been catapulted by a much more powerful special universe. Moreover, the collision of a galaxy with one of two galaxies coming from a different direction [20] reveals the existence of some special universe more powerful than ours. Galaxies in our universe travel along outward radial trajectories and cannot collide among themselves.

One of the stunning discoveries of the last century that still haunts many physicists today is the staggering rate of radial expansion of our universe at accelerated rate $[1,38,39]$. Based on extensive direct measurement of the rate of separation of galaxies from Earth, Edwin Hubble formulated his law that expresses this rate at distance $\mathrm{s}$ kilometers from Earth per kilometer distance:

(4) $\mathrm{d} s / \mathrm{d} t=\rho s$,

where $\rho=1.7\left(10^{-2}\right) / \mathrm{km}$ distance of the receding galaxy from Earth. We measure distance $S$ along a great circle in the spherical dark halo of our universe. Then,

(5) $\mathrm{d} S / \mathrm{d} t=\rho S$.

Since then the estimate of the age of our universe increased from the original 8 billion to the present 14.7 billion and there is talk of raising it to 20 billion. Each time an older star is discovered the estimate is adjusted to accommodate it. This star-chasing game is based on the wrong premise that only our universe ever existed.

Therefore, we stick to the original estimate of 8 billion to solve (4) and find the radius $r$ as function of $t$. Since $\mathrm{d} S / \mathrm{d} t=2 \pi \mathrm{d} r / \mathrm{d} t$ and (5) is independent of the distance between Earth and the other galaxy, it holds when $S=r$. Therefore,

(6) $2 \pi \mathrm{d} r / \mathrm{d} t=\rho r$ or $\mathrm{d} r / r=(\rho / 2 \pi) \mathrm{d} t$.

Solving for $r$, reckoning time from the start of the Big Bang and taking light year and 1 billion years as units of distance and time, respectively, then

$$
\begin{aligned}
& \text { (7) } \left.r(t)=10^{10} \mathrm{e}^{(\rho / 2 \pi)(t-8)} \text { light years (ly }\right), \\
& r^{\prime}(t)=(\rho / 2 \pi) 10^{10} \mathrm{e}^{\rho / 2 \pi(t-8)} \text { ly } / \text { billion years (by), } \\
& r^{\prime \prime}(t)=(\rho / 2 \pi)^{2} 10^{10} \mathrm{e}^{\rho / 2 \pi(t-8)} \mathrm{ly} /(\mathrm{by})^{2}
\end{aligned}
$$

where $10^{10}$ is the present estimate of the radius of our universe [40]. Using standard units we have, at $t=8$,

$$
\begin{aligned}
& \text { (8) } r(8)=3.2\left(10^{22}\right) \mathrm{km} ; r^{\prime}(8)=840 \mathrm{~km} / \mathrm{sec} \text {; } \\
& r^{\prime \prime}(8)=3\left(10^{-10}\right) \mathrm{km} / \mathrm{sec}^{2} .
\end{aligned}
$$

These figures where confirmed recently by Saul Perlmutter, Brian Schmidt and Adam Riess who shared the Nobel Prize for physics in 2011. This accelerated expansion was earlier reported in the scientific literature [38,39].

Since $r^{\prime \prime}>0$, our universe is still approaching its zenith of power. The core of our universe is a tightly packed cocoon shaped cluster of galaxies 650 million years across according to French astronomers. Thus, its destiny is the destiny of its core (super, super massive black hole) plus that of its minor cosmological vortices.

The value of $\rho$ is based on direct observation and analysis of the Doppler effect of a receding light source. Now Encarta Premium has this figure: $\rho=260,000$ $\mathrm{km} / \mathrm{hr} / 3.3$ million light years, i.e., the receding galaxy moves away from Earth faster by $260,000 \mathrm{~km} / \mathrm{hr}$ for every 3.3 million light years distance; it was obviously 
obtained indirectly from records of the past calculated or inferred from data going back at least 3.3 million years ago if they ever existed. Does it make sense?

Converting to standard units and simplifying we have, $\rho=3\left(10^{-19}\right) / \mathrm{km}$ (rate at which a galaxy recedes for every kilometer of distance from Earth). Inserting this value in (7) we obtain, $r^{\prime}(t)=5\left(10^{-14}\right) \mathrm{km} / \mathrm{sec}$, the supposed rate of radial expansion of our universe, and acceleration of $3\left(10^{-32}\right) \mathrm{km} / \mathrm{sec}^{2}$ which point to a static universe that does not match present observation and measurement. Moreover, if it were correct we would have been roasted by intense heat due to the steady formation of stars in the Cosmos and emergence of at least two galaxies discovered since 2004. That would be inconsistent with the average temperature of the Cosmos at $4{ }^{\circ} \mathrm{C}$. Thus, the rise in temperature due to formation of stars and galaxies is offset by our universe's rapid expansion. Finally, we note that there is unbroken nested generalized physical fractal sequence with our universe as first term that goes all the way through the galaxies, stars, planets, moons, cosmic dust, atoms, and superstrings.

\section{The Higgs Boson}

The recent experiment at CERN released powerful burst of energy upon head-on collision of two protons at great speed, each close to the speed of light, and this was attributed to the Higgs boson within the proton. As we have seen the Higgs boson is actually the mini black hole in the eye of the proton. The result of the experiment can be examined to check if one, two or three Higgs bosons were exploded by the collision in the Large Hadron Collider since the two +quarks and the -quarks have their respective mini black holes. The experiment confirms a prediction in [41] that every primal component of coupled primum such as the atom, proton, neutron and neutrino has a mini black hole. When this mini black hole is suitably agitated it converts to huge burst of kinetic energy (prima and photons riding on electromagnetic waves and shock waves). The experiment is also an independent verification of the mini black hole as the source of tremendous energy released by nuclear explosion due to its agitation by nuclear fission which is only a small portion of what is stored in it.

However, an entirely separate but more fundamental question is whether the Higgs boson is indeed God's particle, i.e., the fundamental building block of our universe. To resolve this question, we need to resolve these related questions: 1) How does the Higgs boson fit in and define the structure of the proton and the other elementary particles and the atom? 2) Since the Higgs boson is supposed to endow mass to an elementary particle then its mass must conform to energy conservation, i.e., it has mass. Some advocates of the Higgs boson take the view that it is a number endowed with neither structure nor mass; 3 ) Then how can it give mass to matter?

Thus, there is a fundamental barrier to the Higgs boson being the fundamental building block of matter. Moreover, it is known that in the Cosmos, the cosmological bodies, e.g., stars, galaxies and planets, add up to only $5 \%$ of the mass of our universe. Where is the remaining $95 \%$ ? What is it and how does the Higgs boson fit in it? There is another formidable barrier. One of the two requirements for such fundamental building block is: it must be indestructible; otherwise, our universe would have been unstable and exhausted a long time ago which was not since it has existed for 8 billion years [1] and has even evolved to higher order with the emergence of new natural laws such as biological laws [42]. We have seen its destruction at CERN! The other requirement for the fundamental building block is that every piece of matter is reducible to it which, in effect, would require that there is only one building block, just as there is only one electron replicated at different times and places but having exactly the same composition, structure, behavior and properties. It is not clear if the Higgs boson satisfies this requirement. On a related matter, the claim by advocates that the Higgs boson can explain the origin of our universe is quite a long, long shot. An advocate of the standard model made a similar observation [29]:

"Experimenters will have to verify that the new particle (Higgs boson) is at a spin-0 Higgs boson. Next, they must test how the Higgs boson interacts with other particles to high precision. At this writing its couplings do not quite match predictions, which could be just a statistical fluctuation or a sign of some deeper effect. Meanwhile, experimenters have to keep taking data to see whether more than one Higgs boson exists."

Clearly, this route taken by advocates of the Higgs boson is not easy if at all it leads to it. The author's friendly invitation for them is to take the scientific highroad-GUT - and join him for a grand joint celebration where they will find not the Higgs boson but the superstring as the fundamental building block of matter. The author discovered the superstring in 1997 [6] and quantum physicists the basic units of visible matter, the +quark, -quark and electron, by the 1990s. GUT predicts the existence of their anti-matter and those of the pair of components of the neutrino which are also charged prima [43]. The anti-matter of a negative primum is just beyond the rim of the gravitational flux of the Earth [21]. This information can be verified by experiment.

In a cosmological vortex super massive black hole builds up in the eye so that by the principle of uniformity this is true of every cosmological vortex including a grain of cosmic dust. Again, this is confirmed in $[35,36]$. 


\section{Concluding Remark}

The new and central element in this paper is Qualitative Mathematics and Modeling that shifts the subject matter of science from the appearances of nature to nature itself. It is the methodology that provides the leap from traditional to the new science articulated by GUT. This is the only methodology capable of creating and building physical theory, i.e., a mathematical space whose axioms are laws of nature. Its theoretical applications range from physics, through astrophysics, biology, atmospheric and geological sciences, cosmology and physical psychology and include GUT [1], UTE [42], the Theory of Intelligence $[44,45]$ and Earthly Turbulence $[7,11,46]$. Its practical applications range from engineering through medicine and include research and development on GUT Technologies [47] and design of appropriate technologies for electromagnetic treatment of genetic diseases without side effect [48], a program of creative mathematic science education from primary through graduate school $[49,50]$ and strategy for sustainable development of the Third World [49] based on GUT, UTE, theory of intelligence and theory of turbulence. There are other potential technologies that can be designed based on GUT and UTE aside from those we have presented here. Thus, true to its name, GUT unifies the natural sciences.

Moreover, QMAM is the most appropriate methodology for social science since the latter is not always amenable to computation. We have cited here the development of a program of mathematics-science education program from primary through graduate school as well as the strategy for sustainable development of the Third World Called Strategic Positioning [47,51].

\section{REFERENCES}

[1] E. Escultura, "The Grand Unified Theory," J. Nonlinear Analysis, A-Series: Theory, Methods and Applications (TMA), Vol. 69, No. 3, 2008, pp. 823-831.

[2] E. E. Escultura, "Exact Solutions of Fermat's Equation," Nonlinear Studies, Vol. 5, No. 2, 1998, pp. 227-254.

[3] E. E. Escultura, "Critique-Rectification of Mathematics," In: E. E. Escultura, Ed., Qualitative Mathematics and Modeling: Theoretical and Practical Applications (QMAM: $T P A$ ), in press, LAP LAMBERT Academic Publishing, Saarbrücken, Germany, 2013, pp. 77-129. www.lap-publishing.com

[4] E. E. Escultura, "The New Real Number System and Discrete Computation, Calculus," Neural, Parallel and Scientific Computations, Vol. 17, 2009, pp. 59-84.

[5] E. E. Escultura, "The Mathematics of the Grand Unified Theory," TMA, Vol. 71, 2009, pp. e420-e431.

[6] E. E. Escultura, "The Solution of the Gravitational n-Body Problem," Nonlinear Analysis, A-Series: TMA, Vol. 38, No. 8, 1997, pp. 5021-5032.
[7] E. E. Escultura, "Turbulence: Theory, Verification and Applications," J. Nonlinear Analysis, A-Series: TMA, Vol. 8, 2001, pp. 5955-5966. doi:10.1016/S0362-546X(01)00697-6

[8] E. E. Escultura, "Superstring Loop Dynamics and Applications to Astronomy and Biology," J. Nonlinear Analysis, A-Series: TMA, Vol. 35, No. 8, 1999, pp. 259-285.

[9] E. E. Escultura, "The Trajectories, Reachable Set, Minimal Levels and Chain of Trajectories of a Control System," Ph. D. Thesis, University of Wisconsin, 1970.

[10] H. I. Royden, "Real Analysis," 3rd Edition, MacMillan, New York, 1983.

[11] E. E. Escultura, "Chaos, Turbulence and Fractal: Theory and Applications," International Journal of Modern Nonlinear Theory, in Press. http://www.scirp.org/journal/ijmnta/

[12] Astronomy, August, 1995.

[13] A. Watson, Science, Vol. 281 No. 5375, 1998, pp. 332333.

[14] J. Glanz, Science, Vol. 282, No. 5395, 1998, p. 1806.

[15] V. A. Atsukovsky, "General Ether-Dynamics, Simulation of the Matter Structures and Fields on the Basis of the Ideas about the Gas-Like Ether," Energoatomizdat, Moscow, 1990

[16] G. A. Edgar, "Measure, Topology and Fractal Geometry," Springer-Verlag, New York, 1990. doi:10.1007/978-1-4757-4134-6

[17] E. E. Escultura, "Extended Geometrical and Generalized Fractals, Chaos and Applications," In: K. J. Brennan, Ed., Handbook on the Classification and Application of Fractals, Nova Publishers, 2011, pp. 1-39.

https://www.novapublishers.com/catalog/productin.fophp ?products_id $=23231$.

[18] E. E. Escultura, Journal of Problems of Nonlinear Analysis in Engineering Systems, Vol. 7, No. 1, 2001, pp. 5678.

[19] Hellemans, Science, Vol. 283, No. 5402, 1999, pp. 625626.

[20] Scientific American, Galactic collision, April 1995, pp. 11-14.

[21] E. E. Escultura, "Macro Gravity," In: E. E. Escultura, Ed., QMAM: TPA, in press, LAP LAMBERT Academic Publishing, Saarbrücken, Germany, 2013, pp. 147-161. www.lap-publishing.com

[22] E. E. Escultura, "The Pillars of the New Physics," Nonlinear Studies, Vol. 14, 2007, pp. 241-260.

[23] E. E. Escultura, "Columbia's' Disastrous Final Flight," In: E. E. Escultura, Ed., QMAM: TPA, in press, LAP LAMBERT Academic Publishing, Saarbrücken, Germany, 2013, pp. 281-293. www.lap-publishing.com

[24] E. E. Escultura, "Quantum Gravity,” In: E. E. Escultura, Ed., Scientific Natural Philosophy, Bentham Science Publishers, 2011, pp. 61-80. http://www.benthamscience.com/eBooks/9781608051786 /index.htm

[25] L. Gerlovin, "The Foundations of United Theory of In- 
teractions in a Substance," Energoattomizdat, Leningrad, 1990.

[26] H. A. Nieper, "Revolution in Technology, Medicine and Society," Management Interessengemeinschaft für Tachyon-Feld-Energie, Odenburg, 1 FRG, 1984.

[27] E. E. Escultura, "Qualitative Model of the Atom, Its Components and Origin in the Early Universe," J. Nonlinear Analysis, B-Series: Real World Applications, Vol. 11, 2009, pp. 29-38. doi:10.1016/j.nonrwa.2008.10.035

[28] E. E. Escultura, "The Atom," In: E. E. Escultura, Ed., Qualitative Mathematics and Modeling: Theoretical and Practical Applications (TPA), in press, LAP LAMBERT Academic Publishing, Saarbrücken, Germany, 2013, pp. 144-147. www.lap-publishing.com

[29] R. Garisto and A. Agarwal, Scientific American, Vol. 307, No. 3, September, 2012.

[30] E. E. Escultura, "The Big Bang and What It Was," In: J. R. O'Connell and A. L. Hale, Eds., The Big Bang: Theory, Assumptions and Problems, Nova Science Publishers. https://www.novapublishers.com/catalog/_productinfo.ph p?products_id $=21109$

[31] A. Hellemans, Science, Vol. 282, No. 5382, 1998, pp. 1428-1429; doi:10.1126/science.281.5382.1428b

[32] New Scientist, Cannibalism by giant galaxies, July, 12, 1997, pp. 28-32.

[33] G. Schilling, Science, Vol. 283, No. 5410, 1999, pp. 2013-2014. doi:10.1126/science.283.5410.2003

[34] Encarta Premium, Microsoft Science Encyclopedia, 2008.

[35] Scientific American, The quasars and early galaxies, April 1983, pp. 708-745.

[36] Science, Vol. 283, No. 5401, 1999, pp. 480-481.

[37] E. E. Escultura, J. Nonlinear Analysis, A-Series: TMA, Vol. 63, No. 5-7, 2005, pp. e519-e532.

[38] Glanz, Science, Vol. 282, 1998, p. 5397. doi:10.1126/science.282.5397.2156a

[39] Glanz, Science, Vol. 282, No. 5392, 1998, pp. 1248-1249.

[40] New Scientist, Fractal Universe, June 1999.

[41] E. E. Escultura, "The Higgs Boson," In: E. E. Escultura, Ed., QMAM: TPA, in press, LAP LAMBERT Academic
Publishing, Saarbrücken, Germany, 2013, pp. 218-220.

[42] E. E. Escultura, "The Unified Theory of Evolution,” In: E. E. Escultura, Ed., QMAM: TPA, in press, LAP LAMBERT Academic Publishing, Saarbrücken, Germany, 2013, pp. 161-186. www.lap-publishing.com

[43] E. E. Escultura, "Matter-Anti-Matter," In E. E. Escultura, Ed., QMAM: TPA, in press, LAP LAMBERT Academic Publishing, Saarbrücken, Germany, 2013, pp. 217-218.

[44] E. E. Escultura, "The Theory of Intelligence," In: E. E. Escultura, Ed., QMAM: TPA, in press, LAP LAMBERT Academic Publishing, Saarbrücken, Germany, pp. 187212. www.lap-publishing.com

[45] E. E. Escultura, J. Education and Learning, Vol. 1, No. 12, 2012, pp. 51-64.

http://www.ccsenet.org/journal/index.php/jel/article/view/ view/20095/13268

[46] E. E. Escultura, "Earthly Turbulence,” In: E. E. Escultura, Ed., QMAM: TPA, in press, LAP LAMBERT Academic Publishing, Saarbrücken, Germany, 2013, pp. 265-280.

[47] E. E. Escultura, “GUT Technologies,” In: E. E. Escultura, Ed., QMAM: TPA, in press, LAP LAMBERT Academic Publishing, Saarbrücken, Germany, 2013, pp. 294-335. www.lap-publishing.com

[48] E. E. Escultura, "Electromagnetic Treatment of Genetic Diseases," Open Access J. Biomaterials and Nanobiotechnology, Vol. 3, No. 2, May, 2012, pp. 292-300. http://www.scirp.org/Journal/Home.aspx?IssueID =1673

[49] E. E. Escultura, "Creative Mathematics Education," Open Access J. Creative Education, Vol. 3, No. 1, March 2012, pp. 45-54.

http://www.scirp.org/journal/Home.aspx?IssueID=404 doi: $10.4236 /$ ce. 2012.31008

[50] E. E. Escultura, "Creative Science Education," In: E. E. Escultura, Ed., QMAM: TPA, in press, LAP LAMBERT Academic Publishing, Saarbrücken, Germany, 2013, pp. 316-322.

[51] V. S. R. S. Sarma, E. E. Escultura, Proceedings of International Conference on Sustainable Development, Excel India Publishers, Vol. 1, Chennai, Feb. 1-6, 2010, pp. 241-243. 